\title{
Forecasting of Agricultural Loan in Bangladesh
}

\author{
Md.Mosfiqur Rahman , Masuma Parvin , Sayedul Anam , M.A Rubi
}

\begin{abstract}
The agriculture sector is important to meet up the challenges of twentieth century in Bangladesh. It has huge contribution to our life. This sector secures the food security, export earnings and poverty reduction (Agricultural and MSME finance'2017, BB). In this paper, we forecast the agricultural loan disbursement, overdue and recovery in Bangladesh. Moreover, we have discussed the flaw of loan disbursement, recovery and overdue and that of the way out.
\end{abstract}

Keywords : Time series, ARIMA, ACF, PACF, ADF, Stationary, Autoregressive, Moving average.

\section{INTRODUCTION}

Bangladesh is a country freed in 1971 and after that developing gradually based on agriculture mostly. It has great impact on economy. Majority percent people depend on agriculture directly or indirectly in Bangladesh. The most significant part of gross domestic income comes from the agriculture sector. Currently the contributing rate of agriculture sector to GDP is 14.8 percent and almost 47 percent of labor force employment depending on this sector (Agricultural and MSME finance'2017, BB). Moreover, this sector provides raw material for micro, medium and small industries (Agricultural and MSME finance'2017, BB). So agriculture sector plays a vital role for the development to it's inter connected sector with the remaining part of the economy. Recently the advancement of technology that is introduced to agriculture contributed to its revolutionary production in agriculture sector. But in Bangladesh farmers are not capable to occupy with the advanced technology without the financial support from government as well as private sector. If they are facilitated with the enough financial support it will be easier to uphold the growth of agricultural product in our economy.

As the sector is key fact to achieve the target of self-sufficiency in food production, Bangladesh government has prioritized the agriculture sector. In line with the Bangladesh government, Different Banks and private sectors are making their proactive policy and support to boost up agricultural production. Banks are formulating agricultural loan policy and program accordingly.

Revised Manuscript Received on September 25, 2019

* Correspondence Author

Md.Mosfiqur Rahman, Senior Lecturer in Mathematics, Department of General Educational Development, Daffodil International University, Bangladesh

Masuma Parvin, Senior Lecturer in Mathematics, Department of General Educational Development, Daffodil International University, Bangladesh

Sayedul Anam, Assistant Professor of Mathematics, Department of General Educational Development, Daffodil International University, Bangladesh

M.A Rubi, Lecturer in Statistics, Department of General Educational Development, Daffodil International University, Bangladesh

\section{LITERATURE REVIEW}

The existing procedure in financial institution is procrastinating for the disbursement of loan in Bangladesh. A farmer need to go through a long term process to avail the loan disbursement opportunity in a bank. Long term process in disbursement is a bar to secure agricultural loan. Ruhul Amin Sarker (2006) did a research work where he mentioned that the main impediment in securing loan disbursement is from institutional source recorded by 90 percent farmers. On the other hand bankers are interested to disburse loan to urban areas rather than in rural areas. The ratio of loan-deposit in urban areas is near about 85 percent that is 20 percent more than in rural areas (The Jahangirnagar economic review'2014). There is a shortage of banking operation in rural areas. The banking operation has not spread out adequately in rural areas. As a result the disbursement of agriculture loan from different banks is not quite enough for the farmers especially for medium and large farmers. The argument is accepted by farmers stated a survey (Farmers' credit survey, Sarker et al. 2006; Matias \& Sousa, 2017; Nurgaliyeva et al., 2018). Moreover trivial cooperation has identified as another problems in getting bank loan.

Banking loan rules is one of others obstacle for small and marginal farmers for getting loan. Loan rule are designed very complicatedly that is not apprehended by most of the farmers. A survey conducted by (Ruhul Amin Sarker'2006; Rabbani et al., 2014) showed that 79.2 percent very small farmer in which 82.9 percent identifies the loan rules are difficult to avail the loan from bank and 78 percent of all farmers think same. Alam (1981) categorized four types of non-interested cost of bank loan such as (a) application fees, stamp and documents required in support of loan (b) form filling and writing (c) cost of traveling for loan negotiation (d) cost of entertaining people who assisted in loan negotiation.

To overcome the problems banks should be cooperative and participation among different banks should be ensured. The payment procedure of interest and principal should be readjusted. To increase the recovery rate of loan and to minimize the overdue rate of loan strict supervision is needed very badly from the lender side. Monitoring system must be expanded (Rahman'2010; Rahman'2009). Strict monitoring and supervision is therefore needed by central bank to reduce the tendency of mismanagement in disbursing loan and recovery system. If it is monitored in a proper way overdue of loan will be reduced automatically. Although disbursing rate of loan is increased yearly in amount but it is not enough for increasing demand. After disbursement time loan recovery must be proportional to disbursement to avoid the increasing rate of overdue loan. 


\section{METHODOLOGY}

At the point when a time series isn't stationary, more often than not differencing tasks are connected at the suitable lag with the end goal to accomplish stationary.

\section{RESULTS AND DISCUSSION}

The data that are used in this research is collected from the 'Bangladesh Bank' (https://www.bb.org.bd/pub/publictn.php). In this study, we want to forecast agricultural loan disbursement, recovery and overdue. The yearly data of agricultural loan are given in Data table.

Data Table: Yearly agricultural loan data $(\mathrm{N}[\mathrm{N}]$, 'billion BDT').

\begin{tabular}{|c|c|c|c|}
\hline Year & Disbursement & Recovery & Overdue \\
\hline 2017 & 209.99 & 188.41 & 67.08 \\
\hline 2016 & 176.46 & 170.56 & 56.78 \\
\hline 2015 & 159.78 & 154.07 & 67.29 \\
\hline 2014 & 160.37 & 170.46 & 76.12 \\
\hline 2013 & 146.67 & 143.62 & 52.09 \\
\hline 2012 & 131.32 & 123.59 & 60.52 \\
\hline 2011 & 121.84 & 121.48 & 60.97 \\
\hline 2010 & 111.17 & 101.12 & 64.04 \\
\hline 2009 & 92.84 & 83.77 & 60.8 \\
\hline 2008 & 75.348 & 53.84 & 59.43 \\
\hline 2007 & 52.92 & 46.76 & 66.35 \\
\hline 2006 & 57.89 & 41.24 & 65.99 \\
\hline 2005 & 49.56 & 31.711 & 57.81 \\
\hline 2004 & 40.48 & 31.35 & 62.64 \\
\hline 2003 & 32.78 & 35.16 & 65.26 \\
\hline 2002 & 29.55 & 32.59 & 67.54 \\
\hline 2001 & 30.19 & 28.77 & 67.95 \\
\hline 2000 & 28.51 & 29.96 & 64.58 \\
\hline 1999 & 30.06 & 19.16 & 53.99 \\
\hline 1998 & 16.43 & 16.99 & 54.89 \\
\hline 1997 & 15.17 & 15.94 & 53.12 \\
\hline 1996 & 14.18 & 12.73 & 49.2 \\
\hline 1995 & 14.9 & 11.24 & 44.9 \\
\hline 1994 & 11 & 9.79 & 42.03 \\
\hline 1993 & 8.42 & 8.69 & 38.54 \\
\hline 1992 & 7.94 & 6.62 & 35.72 \\
\hline 1991 & 5.95 & 6.25 & 39.33 \\
\hline 1990 & 6.86 & 7.01 & 32.84 \\
\hline 1989 & 88.07 & 5.78 & 23.55 \\
\hline 1988 & 6.56 & 5.95 & 19.32 \\
\hline 1987 & 6.67 & 11.07 & 15.75 \\
\hline 1986 & 6.31 & 6.07 & 17.78 \\
\hline 1985 & 11.53 & 5.84 & 11.58 \\
\hline 1984 & 10.05 & 5.17 & 7.55 \\
\hline 1983 & 6.78 & 3.42 & 4.56 \\
\hline
\end{tabular}

-Mackinnon (1996) one-sided p-values.

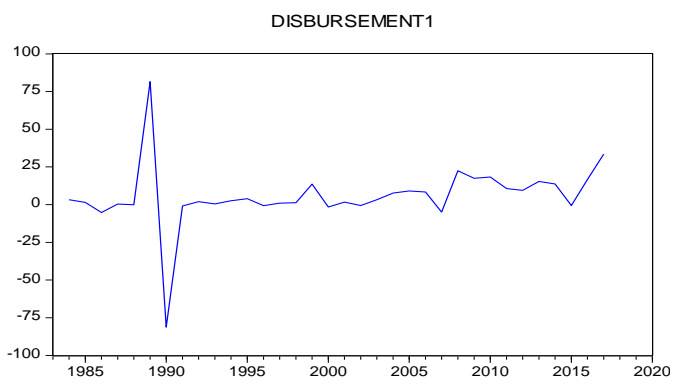

Figure 2: Lag1 of loan disbursement

Table-2: Lag1 table of loan disbursement data Null Hypothesis: DISBURSEMENT1 has a unit root Exogenous: Constant

Lag Length: 0 (Automatic - based on SIC, maxlag=8)

\begin{tabular}{|c|c|c|c|c|}
\hline & & & $\begin{array}{c}\text { t-Statis } \\
\text { tic }\end{array}$ & $\begin{array}{c}\text { Prob. } \\
*\end{array}$ \\
\hline \multicolumn{2}{|c|}{$\begin{array}{c}\text { Augmented Dickey-Fuller test } \\
\text { statistic }\end{array}$} & $\begin{array}{l}-8.061 \\
262\end{array}$ & $\begin{array}{c}0.000 \\
0\end{array}$ \\
\hline $\begin{array}{c}\text { Test critical } \\
\text { values: }\end{array}$ & $1 \%$ & & -3.646 & \\
& level & & 342 & \\
\hline & $5 \%$ & & -2.954 & \\
& level & & 021 & \\
\hline & $10 \%$ & & -2.615 & \\
& level & & 817 & \\
\hline \multicolumn{4}{|c|}{ *MacKinnon (1996) one-sided p-values. } & \\
\hline
\end{tabular}

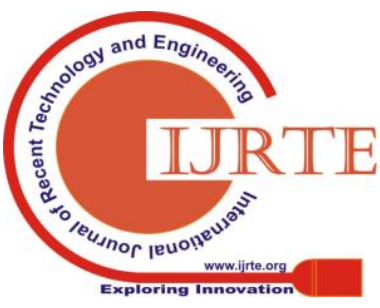


Table-3: Correlogram table for ACF and PC

Date: 10/02/18 Time: $23: 40$

Sample: 19832020

Included observations: 34

\begin{tabular}{|c|c|c|c|c|c|c|c|}
\hline \multicolumn{2}{|c|}{ Autocorrelation } & \multicolumn{2}{|c|}{ Partial Correlation } & $A C$ & PAC & Q-Stat & Prob \\
\hline$\square$ & 1 & $\square$ & 1 & $1-0.361$ & -0.361 & 4.8277 & 0.028 \\
\hline 1 & 1 & 10 & 1 & 20.018 & -0.129 & 4.8403 & 0.089 \\
\hline 1 & 1. & 1 & 1 & $\begin{array}{ll}3 & 0.027\end{array}$ & -0.014 & 4.8696 & 0.182 \\
\hline 10 & 1. & 1 & 1 & $\begin{array}{ll}4 & 0.083\end{array}$ & 0.107 & 5.1506 & 0.272 \\
\hline 1 & 1 & 1 & ב & $\begin{array}{ll}5 & 0.048\end{array}$ & 0.145 & 5.2463 & 0.387 \\
\hline 1 & 1 & 1 & $\sqsupset 1$ & $\begin{array}{lll}6 & 0.067\end{array}$ & 0.176 & 5.4426 & 0.488 \\
\hline 1 & 1 & 1 & 1 & $\begin{array}{ll}7 & 0.023\end{array}$ & 0.139 & 5.4661 & 0.603 \\
\hline 1 & 1 & 1 & 1 & $\begin{array}{ll}8 & 0.027\end{array}$ & 0.093 & 5.4995 & 0.703 \\
\hline 1 & 1 & 1 & 1 & $9-0.030$ & -0.020 & 5.5425 & 0.785 \\
\hline 10 & 1 & 1 & 1 & $\begin{array}{ll}10 & 0.067\end{array}$ & -0.000 & 5.7691 & 0.834 \\
\hline 1 & 1 & 1 & 1 & $11-0.007$ & -0.038 & 5.7716 & 0.888 \\
\hline 1 & 1 & 1 & 1 & $\begin{array}{ll}12 & 0.011\end{array}$ & -0.045 & 5.7783 & 0.927 \\
\hline 1 & 1 & 10 & 1 & $13-0.029$ & -0.080 & 5.8268 & 0.952 \\
\hline 1 & 1 & 10 & 1 & $14-0.032$ & -0.118 & 5.8909 & 0.969 \\
\hline 1 & 1 & 10 & 1 & $15-0.023$ & -0.124 & 5.9259 & 0.981 \\
\hline 1 & 1 & 10 & 1 & $16-0.020$ & -0.115 & 5.9529 & 0.989 \\
\hline
\end{tabular}

Actual and Forecast

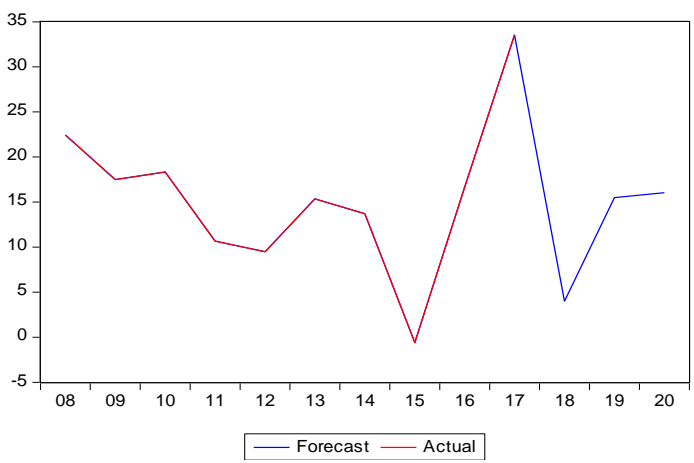

Figure 4: Forecasted graph of loan disbursemen

Automatic ARIMA Forecasting

Selected dependent variable: D(DISBURSEMENT1)

Date: 10/03/18 Time: 21:54

Sample: 19832020

Included observations: 33

Forecast length: 0

Number of estimated ARMA models: 25

Number of non-converged estimations: 0

Selected ARMA model: $(0,2)(0,0)$

AIC value: 7.79177862209

Dependent Variable: D(DISBURSEMENT1)

Method: ARMA Maximum Likelihood (BFGS)

Date: 10/03/18 Time: 21:54

Sample: 19852017

Included observations: 33

Convergence achieved after 43 iterations

Coefficient covariance computed using outer product of gradients

Variable Coefficient Std. Error t-Statistic Prob.

\begin{tabular}{crrrr} 
C & 0.546990 & 0.229916 & 2.379084 & 0.024 \\
& & & -0.01916 & 0.984 \\
MA(1) & -1.678808 & 87.60015 & 4 & 8 \\
& & & & 0.991 \\
MA(2) & 0.678809 & 64.47233 & 0.010529 & 7 \\
SIGMA & & & & 0.983 \\
SQ & 299.3663 & 14659.67 & 0.020421 & 8 \\
\hline \hline
\end{tabular}

\begin{tabular}{l} 
Model Selection Criteria Table \\
Dependent Variable: D(DISBURSEMENT1) \\
Date: $10 / 03 / 18$ Time: $21: 54$ \\
Sample: 19832020 \\
Included observations: 33 \\
\hline \multicolumn{1}{c}{ LogL } \\
\hline \hline
\end{tabular}

Table 4: Regression co-efficient

Hence, the fitted ARIMA $(2,1,0)$ model and the forecasting graph (Fig-4) can be stated as follows: $y_{t}=0.546990-1.678808 \mu_{t}+0.678809 \mu_{t-1}$

\section{The Overdue loan}

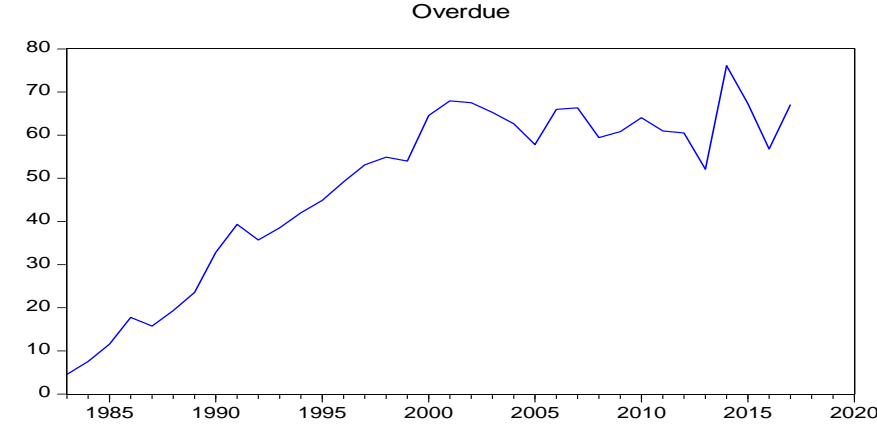

Figure 5: Time graph of overdue loan

Null Hypothesis: OVERDUE has a unit root

Exogenous: Constant

Lag Length: 2 (Automatic-based on $S|C, \max | 9 g=8$ )

\begin{tabular}{lccc}
\hline \hline & t-Statistic & Prob.* \\
\hline \hline Augmented Dickey-Fuller test statistic & -2.886277 & 0.0581 \\
\hline Test critical values: & $1 \%$ level & -3.653730 & \\
& $5 \%$ level & -2.957110 & \\
& $10 \%$ level & -2.617434 & \\
\hline \hline
\end{tabular}

-Mackinnon (1996) one-sided p-values.

Published By: 


\section{Forecasting of Agricultural Loan in Bangladesh}

OVERDUE1

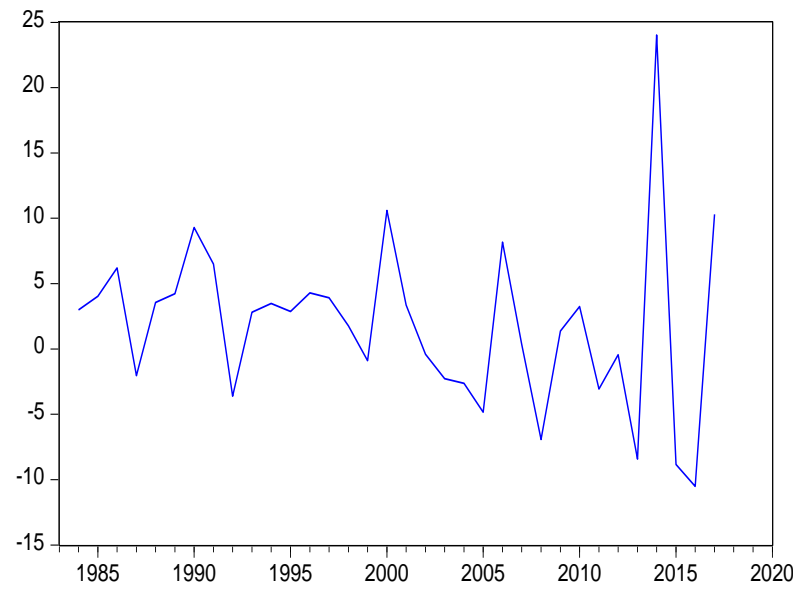

Table 5: Lag1 table of overdue data

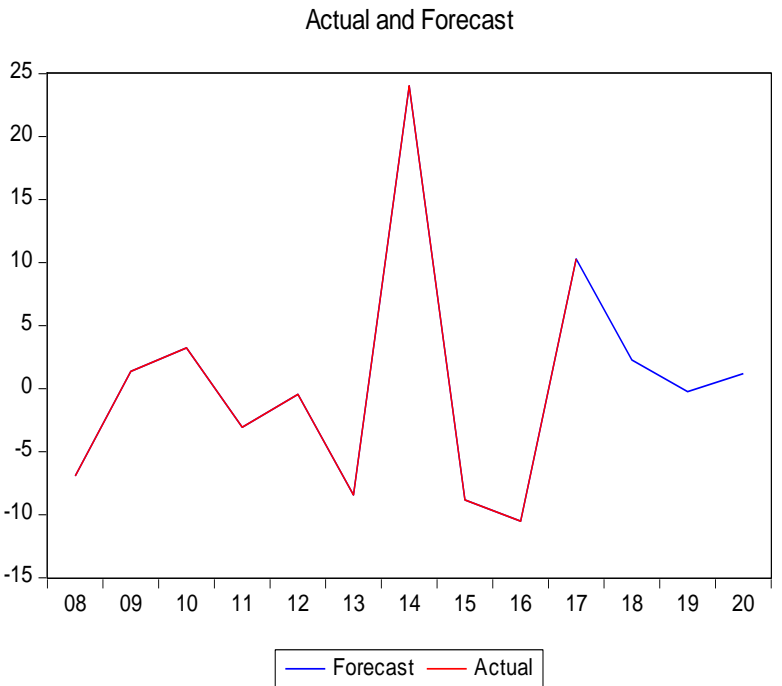

Figure 7: Forecasted graph of overdue loan

Recovery

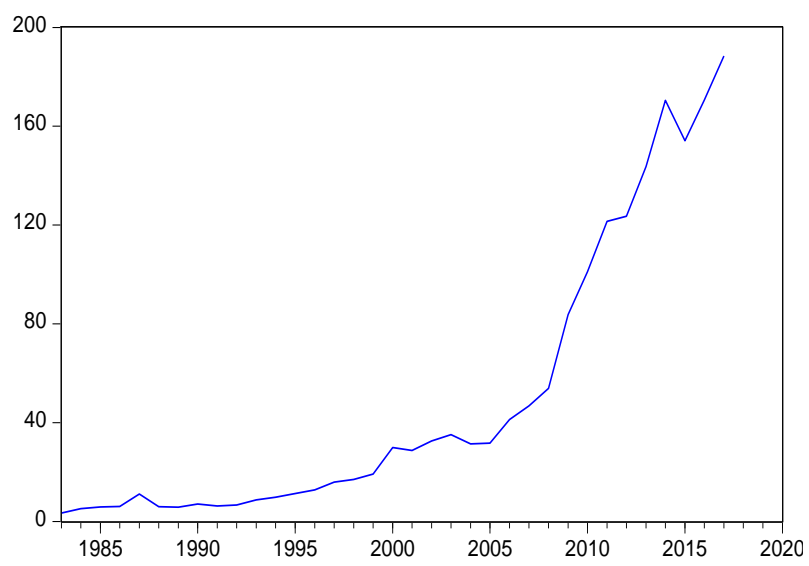

Figure 8: Time graph of loan recovery
Null Hypothesis: RECOVERY has a unit root

Exogenous: Constant

Lag Length: 8 (Automatic - based on $\mathrm{SIC}, \mathrm{mgx} \mid \mathrm{gg}=8$ )

\begin{tabular}{lrrr}
\hline \hline & & t-Statistic & Prob:" \\
\hline \hline Augmented Dickey-Fuller test statistic & 3.161694 & 1.0000 \\
\hline Test critical values: & 1\% level & -3.711457 & \\
& $5 \%$ level & -2.981038 & \\
& 10\% level & -2.629906 & \\
\hline \hline
\end{tabular}

"Mackinnon (1996) one-sided o-values.

RECOVERY1

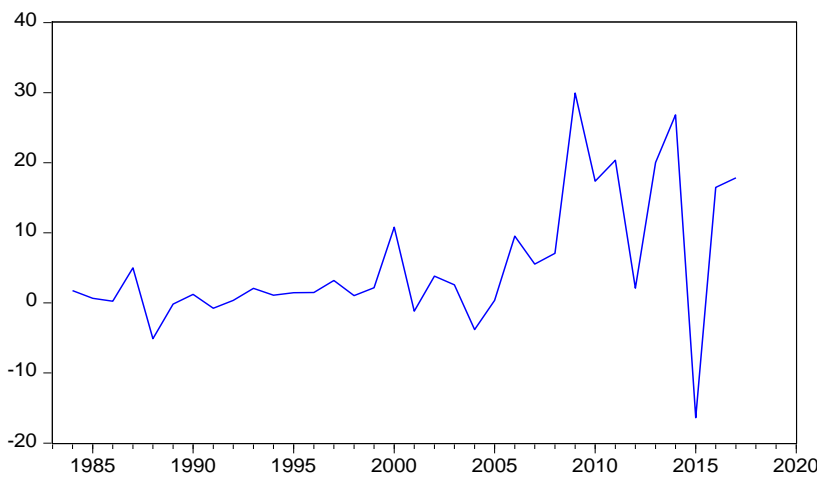

Figure 9: Lag1 graph of loan recovery

Table-8: Lag1 table of loan recovery

Null Hypothesis: RECOVERY 1 has a unit root

Exogenous: Constant

-9g Length: 2 (Automatic-based on SIC, maxlag=8)

\begin{tabular}{lccc}
\hline \hline & & & \\
\hline \hline Augmented Dickey-Fuller test statistic & -1.450644 & 0.5447 \\
\hline Test critical values: & $1 \%$ level & -3.661661 & \\
& $5 \%$ level & -2.960411 & \\
& $10 \%$ level & -2.619160 & \\
\hline \hline
\end{tabular}

-Mackinnon (1996) one-sided p-values.

RECOVERY2

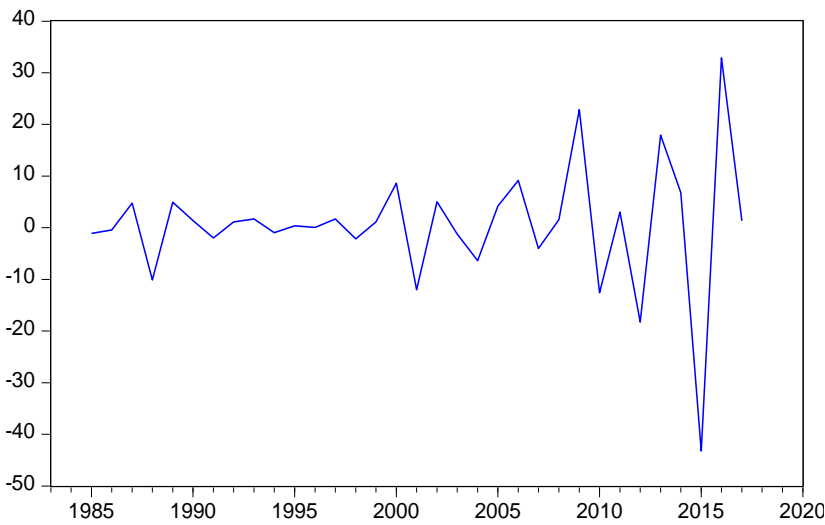

Figure 10: Lag2 graph of loan recovery 
Table-9: Lag2 table of loan recovery

Null Hypothesis: D(RECOVERY2) has a unit root

Exogenous: Constant

Lag Length: 3 (Automatic - based on SIC, maxlag=8)

\begin{tabular}{lccc}
\hline \hline & & $\begin{array}{c}\text { t-Statisti } \\
\text { c }\end{array}$ & $\begin{array}{l}\text { Prob. } \\
*\end{array}$ \\
& & -7.1635 & 0.000 \\
& & 20 & 0 \\
\hline Augmented Dickey-Fuller test statistic & -3.6891 & \\
\hline Test critical & $1 \%$ & 94 & \\
values: & level & -2.9718 & \\
& $5 \%$ & 53 & \\
& level & -2.6251 & \\
& $10 \%$ & 21 & \\
& level & & \\
\hline \hline
\end{tabular}

*MacKinnon (1996) one-sided p-values.

Table-10: Correlogram table for ACF and PC

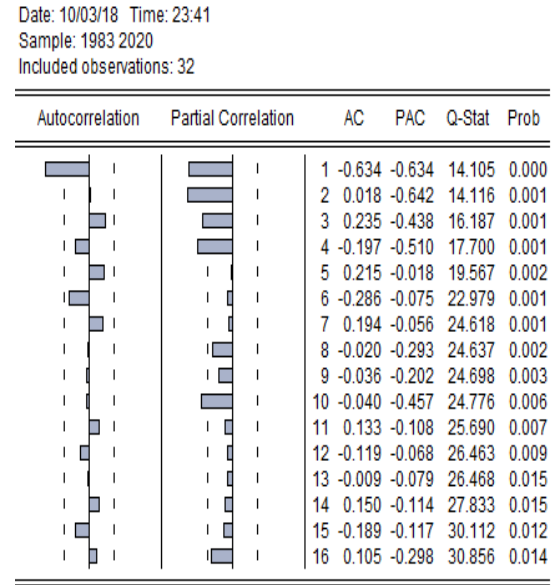

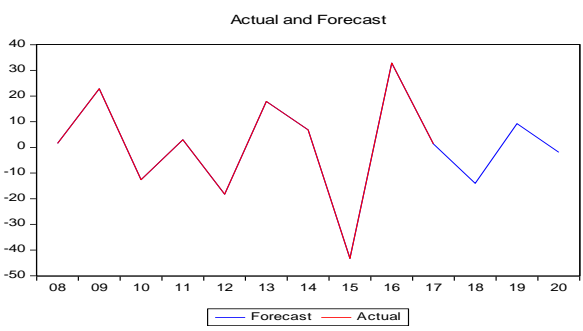

Figure 11: Forecasted graph of loan recovery

Automatic ARIMA Forecasting

Selected dependent variable: RECOVERY2

Date: 10/03/18 Time: 23:47

Sample: 19832017

Included observations: 33

Forecast length: 3

Number of estimated ARMA models: 25

Number of non-converged estimations: 0

Selected ARMA model: $(0,4)(0,0)$
AIC value: 6.59922717625

Table-11: Regression Co-efficient

Model Selection Criteria Table

Dependent Variable: RECOVERY2

Date: 10/03/18 Time: $23: 47$

Sample: 19832017

Included observations: 33

\begin{tabular}{|c|c|c|c|}
\hline Model & $\mathrm{AIC}^{*}$ & $\mathrm{BIC}$ & $\mathrm{HQ}$ \\
\hline$(0,4)(0,0)-109.486476$ & 6.599227 & 6.865858 & 6.691268 \\
\hline$(1,4)(0,0)-109.431207$ & 6.653212 & 6.964281 & 6.760593 \\
\hline$(2,4)(0,0)-109.395721$ & 6.708327 & 7.063835 & 6.831048 \\
\hline$(3,4)(0,0)-109.395703$ & 6.765469 & 7.165415 & 6.903530 \\
\hline$(4,4)(0,0)-109.369187$ & 6.821096 & 7.265482 & 6.974498 \\
\hline$(4,3)(0,0)-111.041799$ & 6.859531 & 7.259478 & 6.997593 \\
\hline$(2,3)(0,0)-113.327944$ & 6.875883 & 7.186952 & 6.983264 \\
\hline$(3,3)(0,0)-112.405763$ & 6.880329 & 7.235837 & 7.003051 \\
\hline$(4,2)(0,0)-112.475388$ & 6.884308 & 7.239816 & 7.007029 \\
\hline$(2,2)(0,0)-114.500041$ & 6.885717 & 7.152348 & 6.977758 \\
\hline$(3,2)(0,0)-113.739504$ & 6.899400 & 7.210470 & 7.006781 \\
\hline$(0.1)(0.0)-117.923824$ & 6.909933 & 7.043248 & 6.955953 \\
\hline$(4,1)(0,0)-114.140221$ & 6.922298 & 7.233368 & 7.029680 \\
\hline$(1.2)(0.0)-116.150865$ & 6.922907 & 7.145099 & 6.999607 \\
\hline$(1,3)(0,0)-115.223300$ & 6.927046 & 7.193677 & 7.019087 \\
\hline$(2,0)(0,0)-117.228006$ & 6.927315 & 7.105069 & 6.988675 \\
\hline$(0,2)(0,0)-117.760686$ & 6.957754 & 7.135508 & 7.019114 \\
\hline$(1,1)(0,0)-117.778835$ & 6.958791 & 7.136545 & 7.020151 \\
\hline$(2,1)(0,0)-117.133348$ & 6.979048 & 7.201241 & 7.055749 \\
\hline$(3,0)(0,0)-117.138665$ & 6.979352 & 7.201545 & 7.056053 \\
\hline$(4,0)(0,0)-117.084432$ & 7.033396 & 7.300027 & 7.125437 \\
\hline$(3,1)(0,0)-117.132356$ & 7.036135 & 7.302766 & 7.128176 \\
\hline$(0,3)(0,0)-119.383690$ & 7.107639 & 7.329832 & 7.184340 \\
\hline$(1,0)(0,0)-124.485528$ & 7.284887 & 7.418203 & 7.330908 \\
\hline$(0.0)(0.0)-129.368412$ & 7.506766 & 7.595643 & 7.537447 \\
\hline
\end{tabular}

Dependent Variable:

RECOVERY2

Method: ARMA Maximum Likelihood (BFGS)

Date: 10/03/18 Time: $23: 47$

Sample: 19852017

Included observations: 33

Failure to improve objective (non-zero gradients) after 76 iterations

Coefficient covariance computed using outer product of gradients

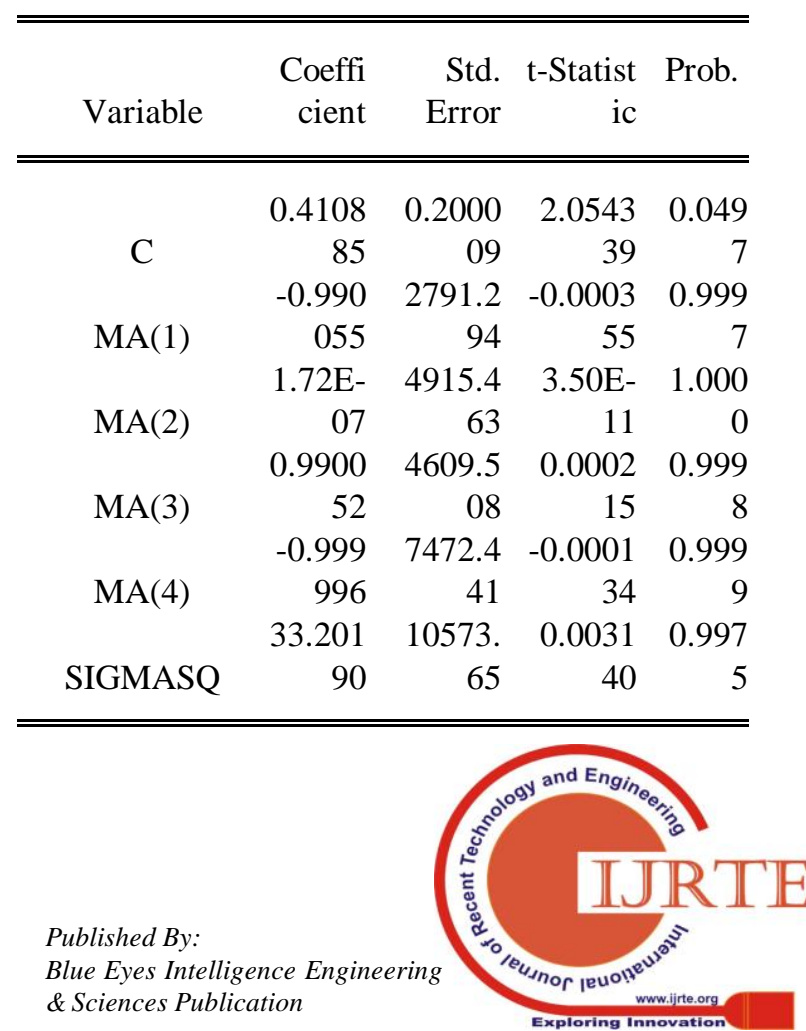




\section{Forecasting of Agricultural Loan in Bangladesh}

Hence, the fitted ARIMA $(4,2,0)$ model and the forecasting graph (Fig-11) can be stated as follows:

$$
\begin{aligned}
& y_{t}=0.410885-0.990055 \mu_{t}+1.72 E-07 \mu_{t-1}+0.99005214 \mu_{t-2} \\
& -0.999996 \mu_{t-3}
\end{aligned}
$$

Finally the fitted ARIMA (2, 1, 0) for the loan disbursement calculated the forecasted loan disbursement that is gradually increasing in amount but the fitted ARIMA $(4,2,0)$ for loan recovery forecasting the declining rate of loan recovery to the disbursement that leads the fitted ARIMA $(3,1,0)$ for overdue rate calculating the forecasted value of overdue rate is scattered.

\section{CONCLUSION}

Exploring the current scenario of agricultural loan in Bangladesh is the main purpose of this study and to identify the liable reason for the growth of agricultural loan in Bangladesh mathematically. Analyzing the data above it is obvious that there are no harmony among loan disbursement, recovery and overdue. Huge difference is observed in between loan disbursement and recovery. Data simulation showing the loan disbursement gradually increasing but at the same time recovery of loan is gradually decreasing. To achieve great success in this sector it is mandatory to keep balance among these factors disbursement, recovery and overdue loan.

\section{ACKNOWLEDGEMENT}

We are thankful to Bangladesh Bank for helping us to collect the data for this research.

\section{REFERENCES}

1. Anam S.,Rahman A., Haque M.M., and Hadi S. R. (2014) "The Impact of Transportation Cost on Potato Price: a Case Study of Potato Distribution in Bangladesh". The International Journal of Management, Vol. 1, No. 03, pp. 01-12, ISSN 2277- 5846.

2. Anam S, Khan, Uddin., "Developing StochasticLinear Programming Model for Production". International Journal of Economic Perspective, 2017, Volume 11, Issue 4, 851-866.

3. Rahman MM, Anam S, Chakrabarty AK, "Forecasting of International Tourists Arrival in Saarc Region and Prospect of Bangladesh". International Journal of Engineering \& Technology(UAE), 7 (3.19) (2018) 161-168. DOI:10.14419/ijet.v7i3.19.17006

4. Raymond Y.C. Tse (1997) "An application of the ARIMA model to real-estate prices in Hong Kong” Journal of Property Finance, Vol. 8, No. 2, pp. $152-163$.

5. Alauddin M, Biswas, "Agricultural Credit in Bangladesh: Trends, Patterns, Problems and Growth Impacts". The Jahangirnagar Economic Review, Vol. 25, June 2014, ISSN 1990-2492.

6. Sarker, Ruhul Amin. 2006. "Rural Financing and Agricultural Credit in Bangladesh: Future Development Strategies for Formal Sector Banks", The University Press Limited, Dhaka.

7. Miah, M. A. Kabir, Alam, A.K.M. Ashraful and Rahman, A.H.M.A. 2006. Impact of Agricultural Credit on MV Boro Rice Cultivation in Bangladesh. Journal of Agriculture and Rural Development 4(1\&2), 161-168.

8. Rahman, M.W, ,Luo, J, and Cheng, E., 2011, "Policies and Performances of agricultural/rural credit in Bangladesh: What is the influence on agricultural production?" African Journal of Agricultural Research, 6(31): 6440-6452 Annual Report, "Bangladesh Bank 2016-2017".

9. I. Altman. Default recovery rates and LGD in credit risk modeling and practice: An updated review of the literature and empirical evidence. Working paper, Stern School of Business, New York University, 2006.
10. Asarnow and D. Edwards. Measuring loss on defaulted bank loans: A 24-year study. Journal of Commercial Lending, 77(7):11-23, 1995.

11. Bellotti and J. Crook. Modelling and predicting loss given default for credit cards.Working paper, Quantitative Financial Risk Management Centre, 2007.

12. Caselli, S. Gatti, and F. Querci. The sensitivity of the loss given default rate to systematic risk: New empirical evidence on bank loans. Journal of Financial Services Research, 34(1):1-34, 2008.

13. Nurgaliyeva, S. A., Zeynolla, S. Z., Galiyeva, A. N., \& Espolova, G. K. (2018). On the issue of modernization of the system of professional development of teachers of high schools of Kazakhstan. Opción, 34(85), 308-326.

14. Dermine and C. Neto de Carvalho. Bank loan losses-given-default: A case study. Journal of Banking and Finance, 30:1291-1243, 2006.

15. Felsovalyi and L. Hurt. Measuring loss on latin american defaulted bank loans: A 27-year study of 27 countries. Journal of Lending and Credit Risk Management, 80:41-46, 1998.

16. Matias, N. R., and Sousa, M. J. (2017). Mobile Health, a Key Factor Enhancing Disease Prevention Campaigns: Looking for Evidences in Kidney Disease Prevention. Journal of Information Systems Engineering \& Management, 2(1), 3. https://doi.org/10.20897/jisem.201703

17. Franks, A. de Servigny, and S. Davydenko. A comparative analysis of the recovery process and recovery rates for private companies in the UK, france and germany. Standard and Poor's report, 2004.

18. Gourieroux, A. Monfort, and A. Trognon. Pseudo-maximum likelihood methods: theory. Econometrica, 52:681-700, 1984.

19. Grunert and M. Weber. Recovery rates of commercial lending: Empirical evidence for german companies. Journal of Banking and Finance, 33:505-513, 2009.

20. Rabbani, M., Bagherzadeh, N., \& Rafiei, H. (2014). Calculating raw material and work-in-process inventories in MTO. MTS production, UCT Journal of Research in Science, Engineering and Technology, 2(3): 109-116.

21. O'Shea, S. Bonelli, and R. Grossman. Bank loan and bond recovery study; 1997-2000.Fitch IBCA, 2001.

22. ]Md. Ariful Islam, Md. Rayhan Islam, Mahmudul Hasan Siddiqui, Luthful Karim. Importance of Agricultural Credit for Rural Development of Bangladesh: A descriptive Approach. International Journal of Business and Economics Research.Vol.2,No.1,2014,pp.68-83.doi:10.11648/j.ijefm.20140201.18 